\title{
Armeria villosa subsp. enritrianoi subsp. nov. (Plumbaginaceae) en el sureste de España (provincia de Jaén)
}

\author{
Gabriel Blanca ${ }^{1}$, Miguel Cueto ${ }^{2}$, Julián Fuentes ${ }^{3}$ \& Salvador Tello ${ }^{4}$ \\ 'Departamento de Botánica. Facultad de Ciencias. Universidad de Granada. 18001 Granada. \\ Departamento de Biología y Geología, CECOUAL, Universidad de Almería. 04120 Almería. \\ ${ }^{3} \mathrm{C} /$ Castillo 5, Bajo F. 18140 La Zubia (Granada). ${ }^{4}$ Paseo del Obispo 7. 23150 Valdepeñas de Jaén (Jaén).
}

\section{Correspondencia}

G. Blanca

e-mail: gblanca@ugr.es

Recibido: 5 junio 2017

Aceptado: 31 octubre 2017

Publicado on-line: diciembre 2017
Armeria villosa subsp. enritrianoi subsp. nov. (Plumbaginaceae) from southern Spain (Jaén province)

Palabras clave: Armeria, Plumbaginaceae, España, Andalucía, provincia de Jaén.

Key words: Armeria, Plumbaginaceae, Spain, Andalusia, Jaén province.
Armeria Willd. (Plumbaginaceae) es un género de gran complejidad taxonómica que incluye alrededor de 80 especies de distribución holártica, con centro de diversidad en el oeste de la Región Mediterránea (Bernis, 1954; Nieto Feliner, 1990a), de manera que el $60 \%$ de los táxones se encuentran en la Península lbérica (Nieto Feliner, 1990a; Fuertes Aguilar et al., 1999).

En la Península Ibérica, con posterioridad a las revisiones de Bernis $(1954,1955,1957)$ y Nieto Feliner (1990a), se han descrito numerosos táxones nuevos (Nieto Feliner, 1987, 1990b; Romo, 1996; Sardinero \& Nieto Feliner, 1997; Nieto Feliner et al., 1998, 2001; Gutiérrez Larena et al., 2004; Crespo Villalba \& Mateo Sanz, 2010; Alejandre Sáenz et al., 2013; Cabezudo et al., 2015), muchos de ellos endémicos de áreas relativamente pequeñas y a menudo con un comportamiento ecológico particular.

Durante un estudio de campo de la Sierra de la Pandera (provincia de Jaén, SE España), detectamos unas poblaciones de Armeria villosa Girard que mostraban claras afinidades con la subespecie más frecuente en territorios adyacentes, $A$. villosa subsp. longiaristata (Boiss. \& Reut.) Nieto Fel., ampliamente distribuida por las montañas Béticas (S España), pero que se distinguían de ésta última por una combinación de caracteres muy particular: hojas anchas con 5-7 nervios, brácteas involucrales externas tan largas como las intermedias, cáliz más pequeño y flores rosadas. Después de consultar las floras regionales más importantes (Willkomm 1868, 1893; Pinto da Silva, 1972; Devesa, 1987; Nieto Feliner, 1990; Salazar, 2011), concluimos que estas poblaciones de la Sierra Pandera constituyen una nueva subespecie, que describimos e ilustramos en este trabajo, y discutimos sus características, distribución y hábitat.

Armeria villosa subsp. enritrianoi Blanca, Cueto, J. Fuentes \& S. Tello, subsp. nov. (figs. 1-2)

Differs from $A$. villosa subsp. longiaristata (Boiss. \& Reut.) Nieto Fel. by having wider (6-16 $\mathrm{mm})$, lanceolate to linear-lanceolate and 5-7-veined leaves, external bracts of the involucre as long as the middle ones, smaller (5.5-7 mm) calyx, and pink corolla.

Holotypus: España, provincia de Jaén, Valdepeñas de Jaén, Sierra de la Pandera, Quiebrajano, Garganta de los Ladrones, 30SVG3565, $860 \mathrm{~m}$ alt., rellanos y repisas de roquedos, en taludes frescos y umbrosos, en terreno calizo-dolomítico, 10-V-2016, G. Blanca, S. Tello \& J. Fuentes (GDA 62619). Isotipos: MA 921490 y MGC 87378.

Etimología. Subespecie dedicada a la memoria del botánico cordobés Enrique Triano (13 Nov. 1967-5 Feb. 2016), que conoció esta planta y apreció sus diferencias con el resto de subespecies de $A$. villosa. 


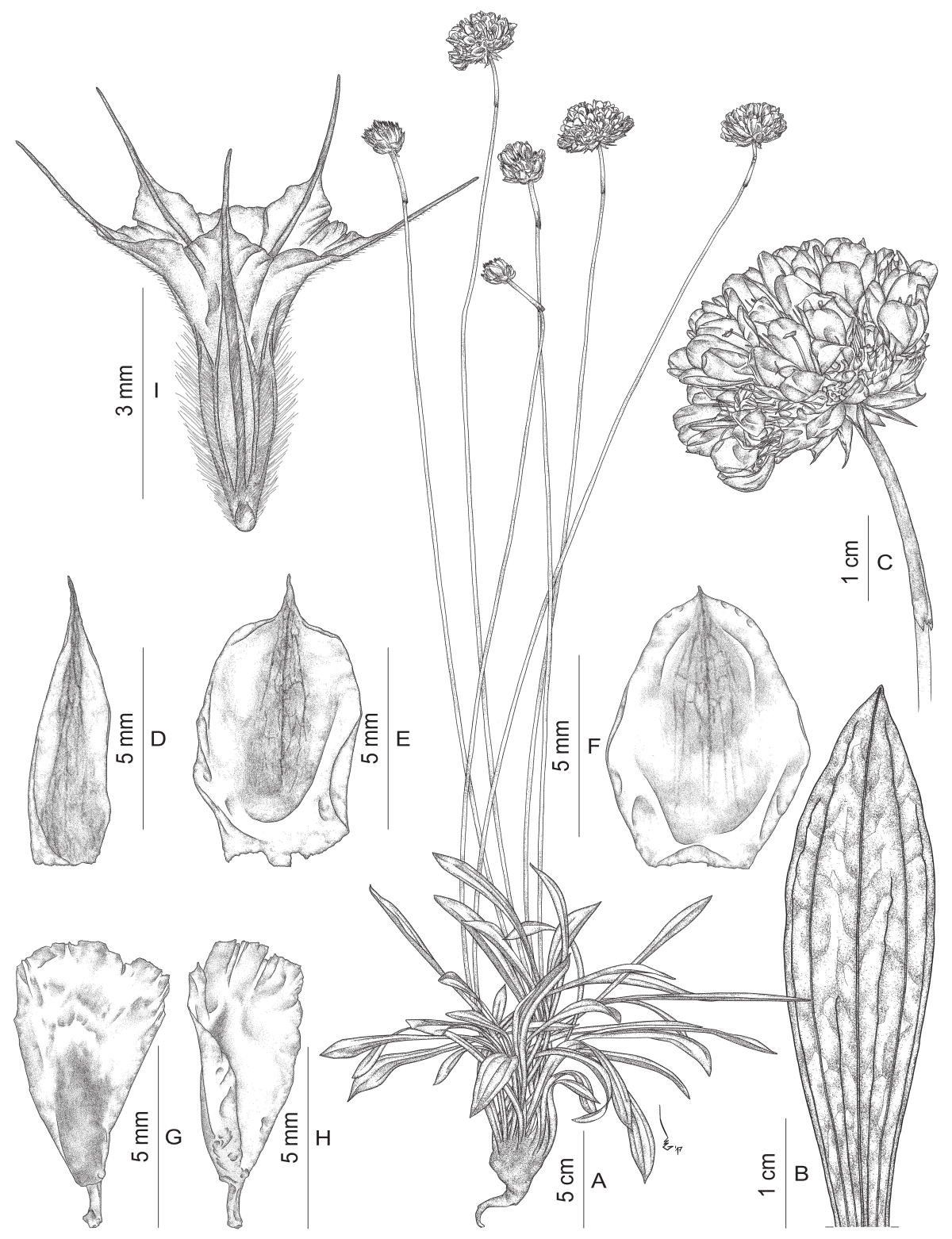

Figura 1. Armeria villosa subsp. enritrianoi: (A) hábito, (B) detalle del limbo foliar, (C) inflorescencia y vaina involucral, (D) bráctea involucral externa, (E) bráctea involucral media, (F) bráctea involucral interna, (G) bráctea espicular (vista dorsal), (H) bráctea espicular (vista lateral), (I) cáliz. Figure 1. (A) habit, (B) leaf limb detail, (C) inflorescence and involucral sheath, (D) outer involucral bract, (E) middle involucral bract, (F) inner involucral bract, (G) spikelet bract (dorsal view), (H) spikelet bract (lateral view), (I) calyx.

Descripción. Hierba perenne, cespitosa, con cepa ramificada y lignificada cubierta de restos foliares en su ápice. Escapos 35-46(-50) cm, erectos. Hojas $50-130^{\prime} 6-16 \mathrm{~mm}$, aglomeradas en la base, homomorfas, lanceoladas o linearlanceoladas, largamente atenuadas hacia la base, con 5-7 nervios, agudas o a veces subobtusas, con granulaciones blancas en el limbo y de margen estrechamente escarioso y ciliado (cilios hasta 0,3 $\mathrm{mm}$ ). Vaina involucral 20-25 mm. Involucro 17-22 $\mathrm{mm}$ de diámetro. Brácteas involucrales 10-14, pajizas, de ápice rugoso teñido de color tostado o verdoso, glabras, las externas tan largas como las intermedias, estrechamente lanceoladas, acuminadas, las intermedias ovadas y cuspidadas, las internas anchamente ovadas o suborbiculares, mucronadas, con margen escarioso ancho. Espículas pediceladas, con brácteas de longitud $\geq$ a las brácteas involucrales internas, en su mayor parte escariosas, con la parte dorsal más consistente y de color verdoso. Cáliz $5,5-7 \mathrm{~mm}$ de longitud (incluyendo las aristas), con las 10 costillas 


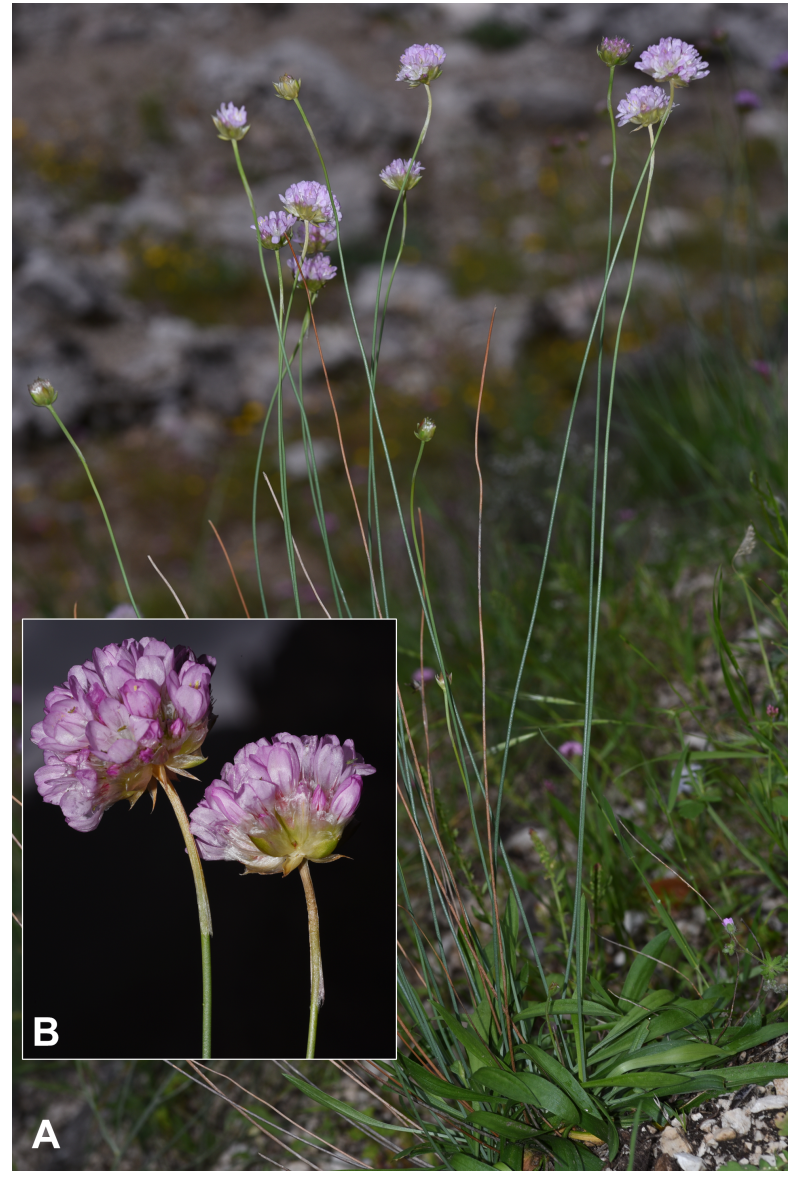

Figura 2. Armeria villosa subsp. enritrianoi: (A) hábito, (B) inflorescencias. Figure 1. (A) habit, (B) inflorescences.

densamente pubescentes (holopleurótico), pelos de 0,2-0,5 mm; espolón c. 0,5 mm; tubo 2,2-2,7 $\mathrm{mm}$; limbo 1,5-1,8 mm, escarioso; lóbulos 2-2,5 $\mathrm{mm}$, largamente aristados. Corola $7-8 \mathrm{~mm}$, de color rosado intenso, con tubo de $2-3 \mathrm{~mm}$, glabro. Filamentos estaminales c. $6 \mathrm{~mm}$, con alas escariosas en la mitad inferior; anteras 1,1-1,3 $\mathrm{mm}$; granos de polen amarillos. Estilos c. $5 \mathrm{~mm}$, con pelos patentes en la base.

Fenología. Florece en mayo y fructifica en junio.

Distribución y ecología. Armeria villosa subsp. enritrianoi es endémica de la Sierra de la Pandera (provincia de Jaén, SE España). Vive en rellanos y repisas de roquedos calizo-dolomíticos, o en zonas pedregosas al pie de los mismos, en lugares frescos y umbrosos, entre 800-1000 m alt.

Observaciones. Armeria villosa subsp. enritrianoi se distingue con facilidad del resto de las subspecies de $A$. villosa por las flores de color rosado. Su aspecto general es parecido a $A$. villosa subsp. longiaristata, que también habita en áreas próximas de la provincia de Jaén, aunque en lugares más abiertos y soleados, pero se distingue sin dificultad porque esta última subespecie presenta brácteas involucrales externas más largas que las medias y a menudo más largas que todas las demás y cáliz más grande $(8,5-11,5 \mathrm{~mm})$. Aún más diferente es la recientemente descrita $A$. villosa subsp. serpentinicola Cabezudo, Pérez Latorre \& Casimiro-Soriguer Solanas, propia de sustratos peridotíticos de la Sierra de Aguas y Sierra de Alpujata (Málaga) (Cabezudo et al., 2015; GaviraRomero et al., 2016), que presenta hojas más estrechas (1,5-7 mm de anchura) y trinervadas, brácteas involucrales de tamaño gradualmente decreciente hacia el interior, y cáliz también más grande $(8-9,5 \mathrm{~mm})$.

El concepto de compiloespecie, propuesto por Harlan \& de Wet (1963) para especies genéticamente "agresivas" que capturan porciones del genoma de otras especies simpátricas por medio de hibridación introgresiva, que se ha sugerido para justificar los modelos de variación ecológica y morfogeográfica en Armeria villosa (Nieto Feliner, 1987, 1988; Fuertes Aguilar et al., 1999; FuertesAguilar \& Nieto Feliner, 2003), puede haber sido el mecanismo que ha actuado en el origen de $A$. villosa subsp. enritrianoi, probablemente por la introgresión de poblaciones de $A$. villosa subsp. longiaristata en otro taxon de corola rosada, durante los periodos de contacto/aislamiento que han ocurrido repetidamente durante los ciclos glaciales/interglaciales del Pleistoceno.

Aplicando las categorías de la Lista Roja de UICN (2012), Armeria villosa subsp. enritrianoi se encuentra en peligro crítico (CR), en base a los criterios B1ab (i,ii, iii) + B2ab (i,ii,iii) + C1.

\section{Otro material estudiado (selección)}

Armeria villosa subsp. enritrianoi Blanca et al.

Jaén: Valdepeñas de Jaén, Sierra de la Pandera, Quiebrajano, $950 \mathrm{~m}$ alt., rellanos y repisas de roquedos, calizo-dolomítico, 10-V-2016, G. Blanca, S. Tello \& J. Fuentes (GDA 62620, paratipo).

\section{Armeria villosa subsp. Iongiaristata (Boiss. \&} Reut.) Nieto Fel.

Albacete: Calar del Mundo, taludes calizos, 1320 m alt., 14-VI-1986, G. Nieto Feliner et al. (MA 403448). Almería: Alicún, yeseras, 11-VI-1971, Fernández Casas (MA 408949); María, Sierra de María, próx. J. B. Umbría de la Virgen, 4-VII-2002, L. Gutiérrez (HUAL 7371); Sierra de Gádor, Llano de la Chanata, 13-VI1996, E. Giménez \& F. Gómez Mercado (HUAL 6246). Cádiz: Serranía de Ronda, Benaocaz, $1000 \mathrm{~m}$ alt., 27VI-1925, Font Quer (MA 145435). Granada: Alamedilla, 
sustrato de origen volcánico, $850 \mathrm{~m}$ alt., 30SVG8263, 16-V-2003, C. Morales et al. (GDA 48095); Cerro Jabalcón, 1450 m alt., 12-VII-1971, Fernández Casas (MA 408852); Cogollos Vega, camino a la Cueva del Agua, 1300 m alt., 30SVG5130, 9-VI-1988, O. Socorro et al. (GDA 21330); Huéscar, Sierra de la Sagra, cortijo de la Umbría, 25-V-1977, A. M. Negrillo (GDA 11706); Montefrío, Sierra de Parapanda, claros de encinar, sustrato calizo, $1250 \mathrm{~m}$ alt., 30SVG1629, 21-V-1989, D. Molina \& M. M. Pérez (GDAC 31905); Pedro Martínez, Mencal, cara N cerro Matías, 1350 m alt., 30SVG8351, 20-V-1990, R. Sánchez Pérez (GDA 26315); Sierra de Alfacar, casas forestales subiendo a Fuente Fría, 21VII-1975, F. Valle (GDAC 9380); Sierra de Loja, prox. Paso de la Cruz, rocas calizas, 900 m alt., 30SUG9512, 2-VI-1989, M. V. García \& M. J. Salinas (GDAC 33215); Sierra de Madrid, Lagunazo, $1250 \mathrm{~m}$ alt., 30SVG2130, 15-VI-1983, P. Aroza et al. (GDA 16002); Sierra Elvira, rocas calizas, 11-V-1968, J. Varo (GDAC 3393); Sierra Nevada, Barranco de los Pasillos, entre el Puerto de la Ragua y Jérez del Marquesado, claros de matorral, 1600 m alt., 30SVG9308, 26-VI-1997, C. Morales \& L. Baena (GDA 47936); Sierra Pera, prox. Cortijo de Pera, 21-V1988, M. A. Varo (GDA 48483); Valle del río Dílar, suelo ácido, quejigal, 1-VI-1996, L. Baena (GDAC 41701). Jaén: Huelma, 3-VI-1927, C. Lacaita (MA 145425); Jamilena, calizas, 1000 m alt., 14-V-1982, C. Fernández (MA 403431); La Iruela, El Cantalar, sustrato calizo, 880 m alt., 12-VI-1982, F. Pugnaire (MA 716500); Sierra de Cazorla, Fuente Acero, 1-VII-1986, M. A. Sevilla \& M. C. Espinar (GDA 18405); Sierra de Mágina, cerro Cárceles, $2000 \mathrm{~m}$ alt., 16-VI-1985, R. Vogt (MA 403428); Sierra de Segura, cerca del Yelmo, pastos calizos bajo el pinar, $1300 \mathrm{~m}$ alt., 14-VI-1986, G. Nieto Feliner et al. (MA 403426); Torredelcampo, barranco de Jabalcuz, VI1976, G. Blanca (GDAC 2984). Málaga: Antequera, El Torcal, 30SUF635925, 6-VI-1989, J. C. de la Rosa \& M. T. Vizoso (GDAC 29397); El Burgo, Sierra Cabrilla, tajos vertiente Norte, calizas, rupícola, paredes de vertisoles a extraplomos, $940 \mathrm{~m}$ alt., 26-V-2010, O. Gavira \& F. Soriguer (MGC 71692); Riogordo/Alfarnatejo, Altos del Fraile, Gomer y Doña Ana, peñas calizas y vaguadas margosas entre ellas, $1000 \mathrm{~m}$ alt., 9-V-2006, A. V. Pérez Latorre et al. (MGC 65146); Ronda, Quejigales, calizas, espinar, $1250 \mathrm{~m}$ alt., 27-VI-1994, A. V. Pérez Latorre et al. (MGC 40036); Sierra de Alfarnate, 26-VI-1919, E. Gros (MA 145441); Valle de Abdalajís, Sierra de Huma, calizas, paredón, orientación Norte, $840 \mathrm{~m}$ alt., 14-VI1996, B. Cabezudo \& A. V. Pérez Latorre (MGC 44200).

\section{Agradecimientos}

Al Dr. Gonzalo Nieto Feliner la lectura crítica del manuscrito y las sugerencias aportadas. A D. Belchí el diseño y realización de los dibujos. A la Consejería de Medio Ambiente y Ordenación del Territorio de la Junta de Andalucía por facilitarnos los permisos para herborizar en territorio andaluz. Nuestra gratitud a los herbarios del Jardín Botánico de Madrid (MA), Universidad de Málaga (MGC), Universidad de Almería (HUAL) y Universidad de Granada (GDA y GDAC).

\section{Referencias}

Alejandre Sáenz, J. A., Barredo Pérez, J. J. \& Escalante Ruiz, M. J. (2013). Armeria castrovalnerana sp. nov., un nuevo taxón de Armeria gr. alpina en el macizo del Castro Valnera (Cordillera Cantábrica, Burgos). Flora Montiberica, 54, 121-135.

Bernis, F. (1954). Revisión del género Armeria Willd. con especial referencia a los grupos ibéricos. Anales del Instituto Botánico Cavanilles, 11, 5-288.

Bernis, F. (1955). Revisión del género Armeria Willd. con especial referencia a los grupos ibéricos. Parte segunda (descriptiva de los grupos ibéricos). Anales del Instituto Botánico Cavanilles, 12, 77-252.

Bernis, F. (1957). Revisión del género Armeria Willd. con especial referencia a los grupos ibéricos. Parte segunda (Conclusión). Anales del Instituto Botánico Cavanilles, 14, 259-432.

Cabezudo, B., Casimiro-Soriguer Solanas, F. \& Pérez Latorre, A. V. (2015). Sobre el género Armeria en la provincia de Málaga (Andalucía, España). Acta Bot. Malacitana, 40, 57-70.

Crespo Villalba, M. B. \& Mateo Sanz, G. (2010). Un nuevo taxón de Armeria (Plumbaginaceae) propio de la Cordillera Ibérica Central. Flora Montiberica, 46, 49-55.

Devesa, J. (1987). Armeria Willd. In B. Valdés, S. Talavera \& E. Fernández-Galiano (Eds.), Flora Vascular de Andalucía Occidental, vol. 1 (pp. 296302). Barcelona: Ketres.

Fuertes-Aguilar, J. \& Nieto Feliner, G. (2003). Additive polymorphisms and reticulation in an ITS phylogeny of thrifts (Plumbaginaceae). Molecular Phylogenetics and Evolution, 28, 430-447.

Fuertes Aguilar, J., Rosselló, J. A. \& Nieto Feliner, G. (1999). Molecular Evidence for the Compilospecies Model of Reticulate Evolution in Armeria (Plumbaginaceae). Systematic Biology, 48, 735-754.

Gavira-Romero, O. N., Hevilla-Ordóñez, S. \& CanoGarcía, E. (2016). Aportaciones a la flora de Sierra Alpujata y Sierra Blanca (Coín, Málaga). Botanica Complutensis, 40, 75-102.

Gutiérrez Larena, B., Fuertes Aguilar, J. \& Nieto Feliner, G. (2004). Morphometric and molecular evidence for taxonomic recognition of a new subspecies of Armeria filicaulis (Plumbaginaceae). Anales del Jardín Botánico de Madrid, 61, 35-48.

Harlan, J. R. \& Wet, J. M. J. de (1963). The compilospecies concept. Evolution, 17, 497-501.

Nieto Feliner, G. (1987). El género Armeria (Plumbaginaceae) en la Península lbérica: Aclaraciones y novedades para una síntesis. Anales del Jardín Botánico de Madrid, 44, 319-348.

Nieto Feliner, G. (1988). Flujo génico en Armeria (Plumbaginaceae) en la Península Ibérica. Lagascalia, 15(suppl.), 233-236.

Nieto Feliner, G. (1990a). Armeria Willd. In S. Castroviejo, M. Laínz, G. López González, P. Montserrat, F. Muñoz Garmendia, J. Paiva \& L. Villar (Eds.), Flora Iberica, vol. 2 (pp. 642-721). Madrid: Real Jardín Botánico, CSIC. 
Nieto Feliner, G. (1990b). Armeria filicaulis subsp. trevenqueana subsp. nov. Anal. Jard. Bot. Madrid, 47, 270-271.

Nieto Feliner, G., Rosselló, J. A. \& Fuertes, J. (1998). A new subspecies of Armeria filicaulis (Plumbaginaceae) from Sierra Nevada (Southern Spain). Anal. Jard. Bot. Madrid, 56, 162-164.

Nieto Feliner, G., Fuertes Aguilar, J. \& Rosselló, J. A. (2001). A new species of Armeria (Plumbaginaceae) from southern Spain with molecular and morphometric evidence on its origin. Botanical Journal of the Linnean Society, 135, 71-84.

Pinto da Silva, A. R. (1972). Armeria Willd. In T. G. Tutin, V. H. Heywood, N. A. Burges, D. M. Moore, D. H. Valentine, S. M. Walters \& D. A. Webb (Eds.), Flora Europaea, vol. 3 (pp. 30-38). Cambridge: Cambridge University Press.

Romo, A. M. (1996). Plantas recogidas por Pius Font
Quer en el Sistema Ibérico. Estudios del Museo de Ciencias Naturales de Álava, 10-11, 195-201.

Salazar, C.(2011). ArmeriaWilld. InG.Blanca, B.Cabezudo, M. Cueto, C. Morales Torres \& C. Salazar (Eds.), Flora VasculardeAndalucía Oriental(pp.610-619). Granada: Universidades de Almería, Granada, Jaén y Málaga. Sardinero, S. \& Nieto Feliner, G. (1997). Una nueva Armeria del Sistema Central (España): $A$. rivasmartinezii (Plumbaginaceae). Collectanea Botanica (Barcelona), 23, 97-104.

UICN (2012). Categorías y criterios de la lista roja de la UICN: Versión 3.1. Segunda edición. Gland, Suiza y Cambridge: UICN.

Willkomm, M. (1868). Armeria Willd. In M. Willkomm \& J. Lange (Eds.), Prodromus Florae Hispanicae, vol. 2 (pp. 362-370). Stuttgart: E. Schweizerbart.

Willkomm, M. (1893). Supplementum Prodromi Florae Hispanicae. Stuttgart: E. Schweizerbart. 